\title{
SEARCH ENGINES IN THE FIELD OF SCIENCE AND TECHNOLOGY: A DOCUMENTATION-CUM-EVALUATION
}

\author{
Shabir Ahmad Rather ${ }^{1}$, Arshad Hussain Bhat ${ }^{2} \&$ Rafiq Ahmad Rather ${ }^{3}$
}

Abstract- This study will attempt to discover most of the search tools existing on the web and to categorize them on the basis of their search features as well as on the nature of their database. Further, the study will try to discover search tools in all science fields particularly life science. Also the tools will be evaluated using a unique evolution method which will help to list them on the basis of their performance in retrieving most relevant information. Later on a search strategy will be designed which will help the web surfers to retrieve a particular information using a particular search tool.

Keywords - Search Engines, Web, Precisian and Recall, Evaluation of Search Engines.

\section{INTRODUCTION}

There is so much proliferation in internet search tools that it is difficult to choose a relevant tool for finding relevant information. Almost in all subjects internet search tools have been designed with different search mechanism. However most of these specific search tools remain discarding as most of the users switch to general type search engines such as Google. Although, Google search engine is most comprehensive search tools as per many research studies. However, it cannot over take a specific search tool developed in a particular field which can provide most relevant information from a small database. Due to continuous research in every field there is information explosion as such more information is being uploaded .Now almost every journal has an online alternative which has proved to be very useful for the information selection as well as for higher generation. However, most of the web surfers are not aware about these developments as such depend only on the limited choices. There has been no attempt so far which would have discovered internet search tools in different subject areas with their search features.Moreever internet search tools follow different search mechanism which gives them popularity over one another. The rest of the Synopses is organized as follows.

\section{SCOPE}

This study will attempt to discover most of the search tools existing on the web and to categorize them on the basis of their search features as well as on the nature of their database.Further, the study will try to discover search tools in all science fields particularly life science. Also the tools will be evaluated using a unique evolution method which will help to list them on the basis of their performance in retrieving most relevant information. Later on a search strategy will be designed which will help the web surfers to retrieve a particular information using a particular search tool.

\section{LITERATURE REVIEW}

The concept of search engines emerged during the last decade of the $20^{\text {th }}$ century. Though the nature of search engines is quite recent, the body of work on web search engines is already quite sizable. Nowadays, growing body of literature is available in print and electronic media which describes various attempts at conducting evaluation of web search engines.

Oppenheim et.al [1] has made a survey of the literature of evaluation of web search engines with the aim of identifying some of the issues and problems in this rapidly dynamic field. The survey shows that research on evaluating search engines has not been carried out in a consistent fashion and as a result it is impossible to compare the performance of search engines as reported by different researchers.

Schwartz [2] has reviewed the way web search engines operate and note the growing body of evaluation literature much of which is not systematic in nature. Another briefly reviews the development of web search engines and consider the current state of affair, and reflects, on their future. The author concludes that performance effectiveness' is difficult to assess in the context of the web. She further suggests a trend towards smaller resource collection with rich metadata and navigation tools.

\footnotetext{
${ }^{1}$ Department of Computer Applications, Government Degree College Anantnag, J\&K, India-192101

${ }^{2}$ Department of Computer Applications, Government Degree College Anantnag, $J \& K$, India

${ }^{3}$ Liberian Government Degree College Anantnag, $J \& K$, India
} 
Londoni,M.and Bells[3] have given a critical and in-depth analysis of information retrieval techniques for evaluation of search engines to highlight the importance of a scientifically sounded approach to search engine evaluation. The authors modify the use of classical evaluation techniques 'relevance', 'recall' and 'precision'.

A set of flexible and proceeding guidelines for the use of these techniques are identified. The authors have recommended some forms of collaboration between IR and web communities to provide a valuable frame work for future studies.

Venditto [4] has tested AltaVista, Info seek, Lycos, Open text WebCrawler and www worm with dozens of search terms over a two-week period. The first 25 results, achieved from a query have seen assessed for relevance testing and effectiveness of search engines by identifying known sites as a particular subject. The author finds that all of the search engines perform well with simple queries but finds differences when compound /complex queries are used.

Shafi and Rather[5] evaluated five search engines pertaining to science, biotechnology and general scope using search terms from the field of biotechnology. The authors focus that the specific search engines possess rich databases but restricted to subscribers. The author prefer general search engines like Google for the users who do not have direct access to the specific databases.

\section{OBJECTIVES}

The Objective of the study is:

1. To identify search engines pertaining to science areas.

2. To evaluate select search engines using precision and recall.

3. To design a search strategy for finding relative information using the select search engines.

4. To find out comprehensiveness and currency of documents retrievable through select search engines.

\section{METHODOLOGY}

There are a number of websites which list search engines in different subjects. These websites will be visited and the search engines pertaining to different subjects will be noted down. However, this will not give total figure of search engines, another method of discovering search engines will be adopted .Google search engine will be used as the main tool to discover search engines pertaining to science subjects. After identification of search engines, there will be a comprehensive evaluative method using search terms. This will help to identify best search engines with their search features and mechanism.

\section{CHAPTERIZATION}

The study will be divided into the following chapters:

Chapter 1. Introduction Reference

Chapter 2.

Search Engines

Reference

Chapter 3.

Review Literature

Reference

Chapter 4.

Analysis of Data

Reference

Chapter 5.

Findings and Discussion

Reference

Chapter 6.

Summary and Conclusion

Reference

Chapter 7.

Bibliography

References 


\section{REFERENCES}

[1] Oppenheim et.al(2000). Progress in documentation : The evaluation of www search engines : Journal of Documentation 56,190-211.

[2] Schwartz [1998].web search engines . Journal of American Society for information sciences , 49,973-982 .

[3] Londoni,M.and Bells(2003).Information retrieval techniques for evaluating search engines: A Critical overview:ASLIB proceedings 52,124-129.

[4] Venditto, G(1998).Search engines showdon. Internetworld,7,79-86.

[5] Shafi,S.M and Rather, Rafiq Ahmad (2005) precision and recall of five search engines for retrieval of scholarly information in the field of Biotechnology: Webology,2(2). 